\title{
NOUVELLE
}

\section{ICSI et signalisation calcique}

Jean-Philippe Wolf, Ahmed Ziyyat, Brigitte Ciapa
Calcium et régulation des gènes en conditions normales et pathologiques

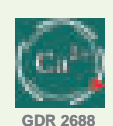

> L'activation de l'ovocyte lors de la fécondation est induite par des variations du niveau intracellulaire de calcium $\left(\mathrm{Ca}_{\mathrm{i}}\right)$ quelle que soit l'espèce considérée. Chez les mammifères dont l'homme, des oscillations de $\mathrm{Ca}_{\mathrm{i}}$ dont l'intensité et la fréquence dépendent de l'espèce, sont non seulement nécessaires et suffisantes pour la reprise de la méiose en métaphase II et l'activation du métabolisme, mais pourraient également contrôler le développement précoce de l'embryon. L'ICSI (intracytoplasmic sperm injection) est reconnue à ce jour comme étant l'approche thérapeutique la plus performante pour pallier une infertilité masculine sévère ou des échecs de FIV (fécondation in vitro). Des résultats obtenus ces quatre dernières années sont susceptibles d'expliquer non seulement les mécanismes à l'origine des oscillations calciques, mais également certains cas d'infertilité pouvant être liés à des défauts de la liaison inter gamétique ou de l'activation ovocytaire, ou d'échecs de fécondation lors de procédures de FIV ou d'ICSI.

L'interaction du spermatozoïde au niveau de la membrane plasmique de l'ovocyte est court-circuitée pendant I'ICSI. Cela conforte l'hypothèse selon laquelle l'activation de l'ovocyte serait due à l'injection d'un (ou de plusieurs) facteur(s) contenu(s) dans le spermatozoïde et possédant les propriétés d'un oscillateur calcique. Cependant, il est possible que certaines voies de signalisation, normalement déclenchées au niveau de la membrane plasmique lors de l'interaction avec le spermatozoïde, ne soient pas activées après ICSI et puissent engendrer des anomalies au cours du développement embryonnaire.
Le signal calcique de la fécondation Dans toutes les espèces animales analysées jusqu'à ce jour, une augmentation rapide et transitoire de la concentration intracellulaire en calcium libre $\left(\mathrm{Ca}_{\mathrm{i}}\right)$ apparaît dans l'ovocyte après fécondation. Ce «signal $C a_{i}$ » est nécessaire et suffisant pour l'activation de l'ovocyte. $\varepsilon n$ effet, son inhibition bloque l'activation de l'œuf fécondé alors que son induction artificielle provoque tous les événements précoces observés lors de la fécondation [1]. Chez l'homme, le signal $\mathrm{Ca}_{\mathrm{i}}$ se présente sous la forme d'oscillations de $\mathrm{Ca}_{i}$, comme pour tous les mammifères [2].

L'ovocyte de mammifère fécondable est bloqué en métaphase de seconde division méiotique. Cet arrêt de la progression du cycle cellulaire est dû au maintien d'activités élevées de MAPK (M-phase activating protein kinase) et de MPF (mitosis promoting factor), le signal $\mathrm{Ca}_{\mathrm{i}}$ généré lors de la fécondation provoquant l'inactivation de ces deux kinases et la sortie de méiose [1]. Des défauts des voies de signalisation, soit à l'origine soit en aval de ce signal, pourraient donc avoir des répercussions sur les événements précoces du développement embryonnaire qui dépendent par exemple de ces deux activités.

Le signal $\mathrm{Ca}_{i}$ est dû essentiellement à une libération de $\mathrm{Ca}_{i}$ du réticulum endoplasmique. Plusieurs voies, non exclusives et pouvant intervenir ensemble lors de la fécondation, ont été proposées pour expliquer comment, à partir du point de fusion avec l'ovocyte, le spermatozoïde déclenche ce signal. À ce jour, aucune n'a été définitivement validée, toute espèce animale confondue (Figure 1) [1, 3]. Certains élé-
J.P. Wolf, A. Ziyyat : Université Paris 13, Laboratoire de Biologie de la Reproduction, UPRES 3410, UFR SMBH, Bobigny, France. AP-HP, Hôpital Jean Verdier, Service d'Histologie, Embryologie et Cytogénétique, Bondy, France. B. Ciapa : UMR CNRS 8080, Développement et Évolution, Université Paris XI, bâtiment 445, 91405 Orsay Cedex, France et GDR 2688.

brigitte.ciapa@u-psud.fr

ments de ces voies situés au niveau de la membrane plasmique sont nécessairement court-circuités après ICSI.

\section{Le signal calcique après ICSI}

Chez l'homme, les oscillations calciques sont observées aussi bien lors de FIV conventionnelles qu'après ICSI [2]. Cependant, alors que les premiers pics calciques apparaissant après ICSI sont tronqués et retardés, les suivants se produisent de façon typique de ceux qui surviennent lors d'une fécondation normale [2]. Lors d'une ICSI, le spermatozoïde, immobilisé puis récupéré dans la pipette d'injection, est injecté dans l'ovocyte en même temps que du cytoplasme de l'ovocyte préalablement aspiré, afin de s'assurer de la rupture de sa membrane plasmique, condition sine qua non du succès de la fécondation. Cette procédure déclenche un influx calcique lequel peut être obtenu après injection de milieu sans spermatozoïde - mais ne provoque alors pas d'activation de l'ovocyte [2]. Cet influx calcique provoqué après ICSI est donc nécessaire mais pas suffisant pour activer l'ovocyte. II serait le signal «déclencheur» normalement activé lors de l'interaction entre les membranes plasmiques du spermatozoïde et de l'ovocyte. Les méthodes d'immobilisation du spermatozoïde utilisées lors de I'ICSI (pipetage, écrasement, application piezo) influencent le démarrage des oscillations $\mathrm{Ca}_{\mathrm{i}}$. Elles endommagent la membrane plasmique du spermato- 
zoïde, condition nécessaire pour libérer soit un facteur spermatique activateur soit des co-facteurs ou des substrats intervenant dans les oscillations $\mathrm{Ca}_{\mathrm{i}}$. La « méthode piezo » est celle qui induit les oscillations $\mathrm{Ca}_{\mathrm{i}}$ le plus rapidement et le

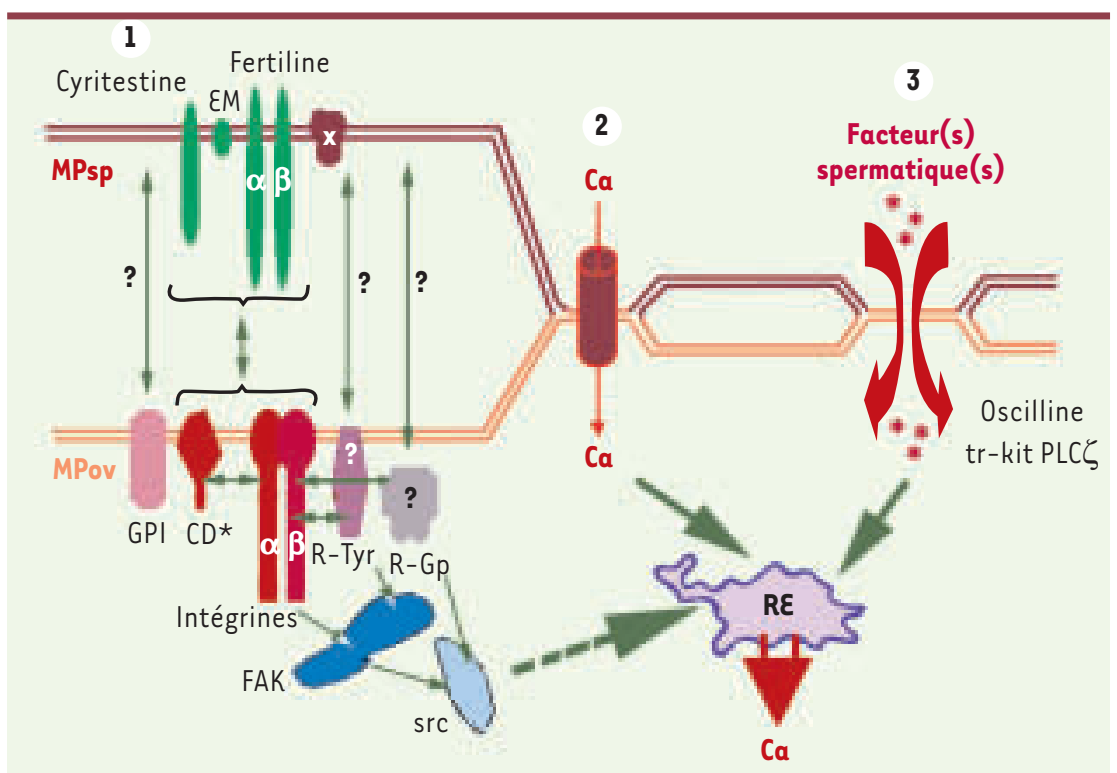

pourcentage de réussite de fécondation le plus élevé [4].

Le décours des oscillations $\mathrm{Ca}_{\mathrm{i}}$ varie en fonction de la maturité de chacun des gamètes utilisés lors de I'ICSI. Les techniques d'ELSI (elongated spermatid injection) et de ROSI (round spermatid injection) conduisent à l'activation de l'ovocyte chez la souris, le hamster et l'homme, l'activation de l'ovocyte après ROSI pouvant se produire sans l'apparition d'oscillations $\mathrm{Ca}_{\mathrm{i}}$ normales [5]. L'ICSI, pratiquée sur des ovocytes maturés in vitro, peut conduire également à des naissances [6]. Le signal $\mathrm{Ca}_{\mathrm{i}}$ pourrait être modifié dans ce cas de façon indirecte par le niveau de MPF qui détermine le temps de formation du pronucléus, lequel régule les oscillations $\mathrm{Ca}_{\mathrm{i}}$ de la fécondation [1].

Enfin, il a été fait état, ces deux dernières années, de naissances obtenues

Figure 1. Les différentes hypothèses d'activation de l'ovocyte lors de la fécondation. 1. Interaction récepteur(s) ovocytaire(s)/ligand(s) spermatique(s). 2. Formation d'un pore entre les gamètes permettant la diffusion dans l'ovocyte d'un (ou de plusieurs) facteur(s) spermatique(s). 3. Insertion dans la membrane plasmique de l'ovocyte de composants de la membrane plasmique spermatique tels que des récepteurs ou des canaux calciques [1, 14]. 1. Des molécules de la membrane plasmique du spermatozoïde (MPsp) peuvent être des ligands de récepteurs de la membrane de l'ovocyte (MPov) : fertiline, membre de la famille des ADAM (A disintegrin and metalloprotease domain) et qui a été clonée chez l'homme, cyritestine ( $A D A M 3)$, éléments de la matrice extracellulaire $(E M)$ comme la fibronectine, ces trois éléments étant des ligands d'intégrines, ou diverses autres molécules $(X)$ telles que des protéines régulatrices du complément dont les récepteurs situés sur l'ovocyte sont inconnus. La mutagenèse dirigée d'ADAM2 (fertiline $\beta$ ) et de la cyritestine (ADAM3) rend infertiles des souris mâles, mais d'autres ADAM, dont le niveau d'expression est réduit dans ces spermatozoïdes, $A d a m 2^{-/-}$ou Adam3 $3^{-/-}$, pourraient être impliquées. Diverses intégrines sont exprimées à la surface de l'ovocyte et leur inhibition avec des anticorps ou des ligands spécifiques inhibent la fécondation, mais leur rôle reste à définir. Des souris femelles invalidées pour les intégrines de la famille $\beta 1$, incluant les sous-unités $\alpha 2, \alpha 3, \alpha 5, \alpha 6, \alpha 9, \alpha v$, ainsi que $\alpha v \beta 3$ et $\alpha v \beta 5$, sont fertiles. Les tétraspanines CD9 et CD81 ( $(C D)$ pourraient aussi être impliquées, des souris femelles $C d 9^{-/-}$produisant des ovocytes incapables de fusionner avec un spermatozoïde, le rôle de CD81 n'ayant pas été clarifié. CD9 et CD81 peuvent interagir avec des intégrines, des récepteurs de protéine $G$ ( $R-G p)$ ou à activité tyrosine kinase (R-Tyr), comme c'est le cas par exemple dans les lymphocytes T. Toutes ces molécules peuvent être intégrées dans un complexe capable non seulement d'induire l'adhésion et la fusion des gamètes, mais aussi de déclencher une voie de signalisation menant au signal $\mathrm{Ca}_{\mathrm{i}}$ via des tyrosine kinases de type FAK (focal adhésion kinase) ou src. 2. Rôle de canaux calciques. Des canaux $\mathrm{Ca}_{\mathrm{i}}$ de la membrane plasmique de l'ovocyte seraient activés lors de l'interaction avec le spermatozoïde, ce qui laisserait passer suffisamment de $\mathrm{Ca}_{i}$ dans l'ovocyte pour stimuler la libération de $C a_{i}$ du RE. Selon une autre hypothèse, la fusion des gamètes entraînerait une insertion de canaux Ca spermatiques au sein de la membrane plasmique de l'ovocyte. Un canal Ca de type TRP-3, exprimé dans le spermatozoïde humain, a été récemment impliqué dans les processus de fécondation chez C. elegans. 3. Le facteur spermatique. Des extraits spermatiques obtenus chez diverses espèces, des invertébrés jusqu'à l'homme, sont capables d'engendrer des oscillations calciques dans les ovocytes de souris, de hamster, humains ou d'oursin ou dans les cellules somatiques. Ce facteur spermatique (étoile) serait donc universel et identique dans toutes les espèces, et soit soluble, soit insoluble et associé au matériel périnucléaire. L'oscilline et tr-kit ont été proposés, le rôle du premier ayant été définitivement invalidé, et aucune preuve définitive n'étayant celui du second.

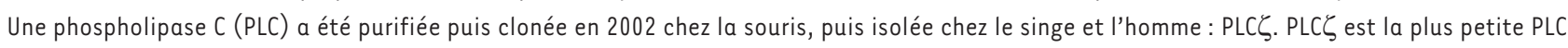
connue à ce jour, montre une sensibilité très élevée au $\mathrm{Ca}$, et induit des oscillations $\mathrm{Ca}_{i}$ exclusivement en phase $\mathrm{M}$ suite à sa localisation nucléaire.

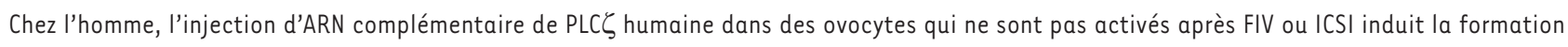

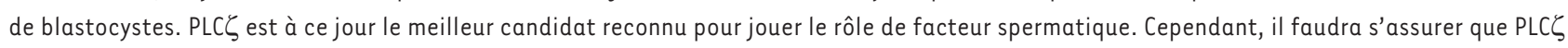

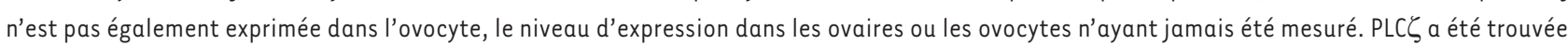
à partir de banques EST (expressed sequence tag) de testicules de souris, ce qui ne correspond qu'à une partie du génome et ne permet en aucun cas

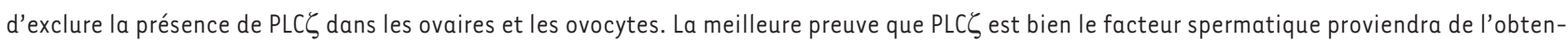
tion de souris infertiles invalidées par délétion de ce gène. 
après ICSI dans des ovocytes pré-activés par du ionophore de calcium $[7,8]$. Cette technique a été pratiquée à la suite d'échecs répétés de fécondation après ICSI classique, mais donc directement chez l'homme sans aucune donnée obtenue au préalable chez la souris ou toute autre espèce animale utilisée en laboratoire. La signalisation $\mathrm{Ca}_{\mathrm{i}}$ dans ces conditions est probablement perturbée, le ionophore de calcium entraînant une augmentation massive de $\mathrm{Ca}_{i}$ secondaire à un apport du milieu extérieur et à une fuite de $\mathrm{Ca}_{i}$ des réserves intracellulaires.

\section{ICSI et empreinte génomique}

Chaque cellule possède deux copies de chaque gène, d'origine maternelle et paternelle, mais il existe des gènes pour lesquels une seule des deux copies s'exprime, l'autre étant réprimée selon l'origine parentale: c'est ce qu'on appelle l'empreinte génomique. Avant la fécondation, un des allèles, maternel ou paternel, est rendu silencieux, puis transmis à la descendance dans cette configuration. Chez l'homme, ce processus est essentiel pour le développement du placenta et de l'embryon. Les mécanismes à l'origine de l'empreinte restent à ce jour mal compris et feraient intervenir des modifications «épigénétiques», c’està-dire non liées à des modifications de la séquence de l'ADN.

L'ensemble du génome subit des modifications épigénétiques lors de la gamétogenèse et pendant le développement embryonnaire selon trois processus: le mutisme des ARN correspondants, des modifications des histones, et la méthylation de I'ADN, celle-ci étant totalement effacée dans les cellules germinales puis ré-établie lors de la gamétogenèse. Après la fécondation, seuls les quelques gènes soumis à l'empreinte parentale restent méthylés. Pour la quasi-totalité du génome, non soumise à l'empreinte parentale, la déméthylation du génome paternel est activée dans les premières heures suivant la fécondation, puis elle est suivie d'une déméthylation passive du génome maternel. L'ensemble du génome est ensuite re-méthylé lors du développement embryonnaire [9]. Les mécanismes à l'origine de la déméthylation active du génome paternel après la fécondation, ainsi que ceux protégeant le génome maternel à ce tout début de l'embryogenèse, sont mal définis à ce jour.

Des défauts épigénétiques peuvent conduire à des pathologies majeures incluant des cancers, divers syndromes liés à des instabilités chromosomiques, et des retards mentaux. Des perturbations de l'empreinte parentale ont aussi été associées à certaines maladies, notamment au cancer [10]. Une anomalie de la répression des deux copies d'un gène contrôlant la prolifération cellulaire peut en effet contribuer à la genèse de certaines tumeurs. Des défauts de l'empreinte génétique d'une région du chromosome 15 conduisent aux syndromes de Prader-Willi ou d'Angelman qui sont associés à divers problèmes de développement et de comportement, et mentaux. Or, une augmentation possible de la fréquence de l'apparition de ces deux syndromes chez des enfants conçus par fécondation assistée et plus précisément après ICSI a été suggérée, le nombre de ces cas rapportés restant cependant insuffisant pour en tirer une conclusion [11]. En revanche, un risque accru de développement du syndrome de Beckwith-Wiedemann a été démontré chez les enfants conçus par FIV. Ce syndrome est associé à des anomalies épigénétiques dans un cluster de gènes soumis à l'empreinte dans une région du chromosome 11 et entraîne une macrosomie et un risque de néoplasie augmenté [12]. De même, il a été rapporté une augmentation de la fréquence des anomalies graves chez les enfants issus de FIV et des nouveau-nés à petits poids de naissance sans qu'il soit possible de savoir, pour le moment, si le risque est lié à la FIV ou à la cause de l'infertilité des parents [13].

\section{Conclusions}

Les voies de signalisation issues de la membrane plasmique et activées par l'interaction et la fusion entre les gamètes sont courtcircuitées lors de l'ICSI. Un chaînon ou un élément manquant peut-il avoir des répercussions sur les processus épigénétiques? La déméthylation active du génome paternel est-elle liée à la signalisation calcique lors de la fécondation? Des défauts des oscillations calciques, ou des signaux calciques aberrants, par exemple issus de l'activation préalable de l'ovocyte par du ionophore au calcium comme mentionné ci-dessus, peuvent-ils avoir une incidence sur les mécanismes d'épigenèse stimulés après fécondation? L'ICSI est pratiquée depuis 1992, et aucun des enfants n'a donc encore à ce jour atteint l'âge adulte. Or, certains cancers et certaines maladies neurologiques ou musculaires ne se révèlent qu'à l'âge adulte. La sécurité vis-à-vis de l'empreinte génétique des techniques utilisées en FIV demanderait à être évaluée avec attention. $\diamond$

ICSI and calloium signaling

\section{RÉFÉRENCES}

1. Malcuit C, Kurokawa M, Fissore RA. Calcium oscillations and mammalian egg activation.J Cell Physiol 2006; $206: 565-73$.

2. Tesarik J. Calcium signalling in human oocytes and embryos: two-store model revival. Hum Reprod 2002; $17: 2948-9$.

3. Santella L, Lim D, Moccia F. Calcium and fertilization: the beginning of life. Trends Biochem Sci 2004 ; $29: 400-8$.

4. Yanagida K, Katayose $\mathrm{H}$, Hirata S, et al. Influence of sperm immobilization on onset of $\mathrm{Ca}^{2+}$ oscillations after ICSI. Hum Reprod 2001 ; 16 : 148-52.

5. Yazawa H, Yanagida K, Katayose $H$, et al. Comparison of oocyte activation and $\mathrm{Ca}^{2+}$ oscillation-inducing abilities of round/elongated spermatids of mouse, hamster, rat, rabbit and human assessed by mouse oocyte activation assay. Hum Reprod 2000 ; 15 : 2582-90.

6. Liu J, Lu G, Qian Y, et al. Pregnancies and births achieved from in vitro matured oocytes retrieved from poor responders undergoing stimulation in in vitro fertilization cycles. Fertil Steril 2003; 80 : 447-9.

7. Chi HJ, Koo JJ, Song SJ, et al. Successful fertilization and pregnancy after intracytoplasmic sperm injection and oocyte activation with calcium ionophore in a normozoospermic patient with extremely low fertilization rates in intracytoplasmic sperm injection cycles. Fertil Steril $2004 ; 82$ : 475-7.

8. Murase $Y$, Araki $Y$, Mizuno S, et al. Pregnancy following chemical activation of oocytes in a couple with repeated failure of fertilization using ICSI: case report. Hum Reprod 2004 ; 19 : 1604-7.

9. Kelly TL, Trasler JM. Reproductive epigenetics. Clin Genet $2004 ; 65: 247-60$.

10. Paoloni-Giacobino A, Chaillet JR. Genomic imprinting and assisted reproduction. Reprod Health $2004 ; 1: 6$.

11. Gosden R, Trasler J, Lucifero D, Faddy M. Rare congenital disorders, imprinted genes, and assisted reproductive technology. Lancet $2003 ; 361$ : 1975-7.

12. Maher $\varepsilon R$, Brueton LA, Bowdin SC, et al. BeckwithWiedemann syndrome and assisted reproduction technology (ART). J Med Genet 2003; $40: 62-4$.

13. Schieve LA, Meikle SF, Ferre C, et al. Low and very low birth weight in infants conceived with use of assisted reproductive technology. N Engl J Med 2002 ; 346 : 731-7.

14. Stein KK, Primakoff P, Myles D. Sperm-egg fusion: events at the plasma membrane. J Cell Sci 2004 . $117: 6269-74$. 